\title{
Glycation of whey protein with dextrans of different molar mass: Effect on immunoglobulin E-binding capacity with blood sera obtained from patients with cow milk protein allergy
}

\author{
Lei Xu, ${ }^{*}$ Yuansheng Gong, ${ }^{*}$ James E. Gern, $\dagger$ Shinya Ikeda, ${ }^{*}$ and John A. Lucey ${ }^{*}{ }^{1}$ \\ *Department of Food Science, \\ †School of Medicine and Public Health, and \\ ¥Center for Dairy Research, University of Wisconsin, Madison 53706
}

\begin{abstract}
A growing concern around the world is the number of people who are suffering from food protein allergies. One potential approach to decrease protein allergenicity is to block IgE-binding epitopes of the protein allergen by attachment of polysaccharides via the Maillard reaction (i.e., glycation). Protein glycation has been extensively studied to modify various functional properties. We wanted to examine whether glycates could reduce $\operatorname{IgE}$ binding in patients with cow milk protein allergy and to explore how the size (molar mass; $M_{W}$ ) of the polysaccharide affects this IgE-binding capacity. Glycation was performed using the initial step of the Maillard reaction performed in aqueous solutions. The specific goal of this study was to reduce the IgEbinding capacity of whey protein isolate (WPI) through glycation with dextran (DX). Blood sera were obtained from 8 patients who had been diagnosed with cow milk protein allergy, and a composite sera sample was used for IgE-binding analysis by the ImmunoCap (Phadia, Uppsala, Sweden) method. The WPI was glycated with DX of $M_{W}$ ranging from 1 to $2,000 \mathrm{kDa}$, and the $M_{W}$ of purified glycates was determined using size-exclusion chromatography coupled with multiangle laser light scattering. The WPI to DX molar ratios in the glycates made from DX that had $M_{W}$ values of $1,3.5,10$ (G10), 150,500 , and 2,000 $\mathrm{kDa}$ were 1:4, 1:3, 1:2, 1:1.5, 1:1, and $1: 1$, respectively. With the increase in the $M_{W}$ of DX, there was an increase in the $M_{W}$ values of the corresponding glycates but a decrease in the number of bound DX. The WPI-DX glycates had lower whey protein IgE-binding capacity than native WPI, with the lowest IgE-binding capacity obtained in the G10 glycate. The DX binding ratios and morphology results from atomic force microscopy images suggested that
\end{abstract}

Received December 22, 2017.

Accepted April 11, 2018.

${ }^{1}$ Corresponding author: jlucey@cdr.wisc.edu glycation of WPI with small- $M_{W}$ DX resulted in extensive protein surface coverage, probably due to the attachment of up to $4 \mathrm{DX}$ molecules per whey protein. The lower IgE binding of the G10 glycate was likely due to greater steric hindrance (or a physical barrier) at the surface of the protein. In summary, our results demonstrate that glycating WPI with DX via Maillard reaction can potentially be used to decrease the allergenicity of whey protein.

Key words: dextran, whey protein isolate, Maillard reaction, cow milk protein allergy, proteinpolysaccharide glycates

\section{INTRODUCTION}

Cow milk protein allergy (CMPA) is a common food protein allergy, especially in infants, with the reported incidence ranging from 0.1 to $7.5 \%$ globally, although the incidence significantly decreases in children older than 3 yr (Wal, 2002). Cow milk protein allergy is commonly defined as an immunologically mediated reaction that could be $\operatorname{IgE}$ or non- $\mathrm{IgE}$ associated and is triggered by a potential sensitizing antigen (protein) in cow milk. Various types of milk proteins (both caseins and whey proteins) have been implicated in this reaction (Wal, 2002). Milk-based infant formula is usually formulated with a high proportion of whey proteins. Reducing the allergenicity of whey proteins could expand their food and nutritional uses. Established dietary treatments for infants with CMPA include protein hydrolysis and the use of nondairy infant formula (e.g., soy formula; Dupont and De Boissieu, 2003). Extensive protein hydrolysis results in poor physical and functional properties as well as undesirable bitterness in the formula.

Protein functionality can also be modified through glycation, with reducing sugars or polysaccharides (for reviews, see Hattori, 2002; Liu et al., 2012; O'Mahony et al., 2016). This glycation process is usually performed by dry heating (e.g., with lyophilized samples) exploiting the initial step in the Maillard reaction. Glycation 
has been exploited to improve protein solubility, heat stability, and emulsification. However, dry heating processes are not feasible from an industrial perspective (de Oliveira et al., 2016). In addition, dry heating results in the formation of unwanted color and flavor compounds as well as extensive protein denaturation and aggregation. Zhu et al. (2008) developed a novel alternative method in which glycation was performed in an aqueous (wet) environment but using high concentrations of dextran (DX) as a crowding agent. Macromolecular crowding is the presence of high concentrations $(>20 \%)$ of macromolecules in a solution; this important phenomenon is well recognized in various biological systems, such as cells (Ellis, 2001). Crowding enhances the excluded volume effect, which has the benefit of reducing the opportunity for (unwanted) protein unfolding and denaturation. With the aqueous approach, the glycation reaction can be continuously monitored by UV spectroscopy and stopped before there is unwanted color formation.

Cow milk proteins have been glycated with various sugars and polysaccharides by the dry heating method as well as chemical treatments to try to reduce their immune response (as assessed mostly by indirect methods; Hattori et al., 2000, 2004; Kobayashi et al., 2001; $\mathrm{Bu}$ et al., 2009; Taheri-Kafrani et al., 2009; CorzoMartínez et al., 2010; Nodake et al., 2010; Li et al., 2011; Zhang et al., 2014). Corzo-Martínez et al. (2010) used dry heating to glycate individual whey proteins with galactose, tagatose, and small molar mass $\left(\boldsymbol{M}_{W}\right)$ DX, but no significant reduction in $\operatorname{IgE}$ binding was observed for these samples. However, in that study the glycates were not purified before IgE testing (CorzoMartínez et al., 2010). Immunoglobulin E-binding capacity is an important characteristic of food allergens and is commonly used as a measure for the evaluation of the safety of new foods, recommended in the guidance documents published by FAO/WHO (2001) and EFSA (2004) for the safety assessment of novel foods and particularly foods derived from biotechnology. In this research, the IgE-binding capacity was evaluated by the ImmunoCAP (Phadia, Uppsala, Sweden) test, one of the standard tools for in vitro allergy diagnostics in American hospitals, and approved by the US Food and Drug Administration up to a detection limit of 0.1 kUA/L (Johansson, 2004).

The goal of this research was to reduce the IgE-binding capacity of whey protein isolate (WPI) by glycating it with DX. Initial work by our group indicated that WPI glycated with DX (10 kDa) via the aqueous method could reduce IgE binding (Böttger, 2013). However, the effect of the $M_{W}$ of DX on reducing whey protein $\mathrm{IgE}$ binding was not investigated. During this study, the effect of the $M_{W}$ of $\mathrm{DX}$, ranging from 1 to $2,000 \mathrm{kDa}$, on the properties of WPI-DX glycates was explored. The $M_{W}$ of purified glycates was evaluated with sizeexclusion chromatography coupled with multiangle laser light scattering (SEC-MALLS). Dextran has been successfully used in the past to produce individual whey protein glycates by the dry heating method (e.g., Jiménez-Castaño et al., 2007) and WPI by the aqueous method (Zhu et al., 2008, 2010). Previous studies about the effect of the $M_{W}$ of polysaccharide on whey proteinpolysaccharide glycates mainly focused on functionality improvements, such as emulsion stability (Dunlap and Côté, 2005), foaming properties (Ter Haar et al., 2011), heat stability (Shu et al., 1996), and other physical properties (Kato, 2002), rather than how different $M_{W}$ polysaccharides affect IgE binding. There does not appear to be previous studies on the IgE-binding capacity of various sizes of whey protein glycates prepared by the aqueous heating method. Some previous studies on glycates prepared with the dry heating method did not purify glycates from the reaction mixture; thus, it was not clear whether the $\operatorname{IgE}$ results were actually due to glycation or could be caused by extensive protein denaturation (which can also reduce allergenicity). In the current study, purified glycates were tested by incubation with sera from blood samples obtained from patients with CMPA (as this is a more direct reflection of allergenicity than indirect chemical tests).

Our hypothesis was that glycation of WPI with DX would shield the epitopes on the allergen (whey proteins) by creating steric hindrance (physical barrier) on the protein, thus limiting the accessibility of IgEbinding sites. After characterizing the $M_{W}$, morphology, and WPI to DX binding ratio of the glycates, we evaluated the whey protein-specific IgE-binding capacity of glycates compared with the native protein (WPI). We also evaluated the microstructure and morphology of glycates by atomic force microscopy (AFM) to help explore the possible mechanism by which these polysaccharides may reduce IgE binding.

\section{MATERIALS AND METHODS}

\section{Materials}

For glycation we used WPI from Davisco Foods International Inc. (Le Sueur, MN), which had a total protein content of $>95 \%$ (dry basis) and a lactose content of $<1 \%$. The WPI was thoroughly dialyzed against MilliQ water (Millipore Corp., Billerica, MA) for $3 \mathrm{~d}$ at $5^{\circ} \mathrm{C}$, with the water changed every $6 \mathrm{~h}$ to remove residual lactose and minerals. Dialysis membrane tubing had a $M_{W}$ cutoff of 6,000 to 8,000 Da (Spectrum Laboratories Inc., Rancho Dominguez, CA). After lyophilization, purified WPI powders were stored at $5^{\circ} \mathrm{C}$. 
Dextran from Leuconostoc mesenteroides with small $M_{W}$ (reported $M_{W}$ of $1,3.5$, and $10 \mathrm{kDa}$ ) was obtained from Pharmacosmos (Holbaek, Denmark). Dextran with larger $M_{W}$ (reported $M_{W}$ of 150,500 , and 2,000 $\mathrm{kDa}$ ) was purchased from Sigma (St. Louis, MO). Before use, all DX were dialyzed against Milli-Q water using a dialysis membrane with different $M_{W}$ cutoff to remove low-molecular-mass oligosaccharides.

Blood sera from 8 patients who were clinically diagnosed as having CMPA were provided by the University of Wisconsin-Madison Department of Medicine, Division of Allergy and Clinical Immunology. Sera were obtained from children who participated in the COAST (Childhood Origins of Asthma) study.

\section{Preparation of WPI-DX Glycates}

The WPI was glycated with DX with different $M_{W}$ : $1 \mathrm{kDa}$ (DX1), $3.5 \mathrm{kDa}$ (DX3.5), $10 \mathrm{kDa}$ (DX10), 150 $\mathrm{kDa}, 500 \mathrm{kDa}$, and 2,000 kDa. The glycation protocol was based on that developed by Zhu et al. (2008, 2010); we evaluated the effect of various incubation times (from 7 to 48 h). The WPI (10\%,wt/wt) and DX (30\%,wt/ wt) were rehydrated in $10 \mathrm{~m} M$ sodium phosphate buffer (SPB; pH 6.5), and the mixtures were stirred overnight at $5^{\circ} \mathrm{C}$ to ensure complete hydration. Following pH adjustment to 6.5 , samples were incubated in a water bath at $62^{\circ} \mathrm{C}$ for 7 (glycates made from DX that had an $M_{W}$ value of $1 \mathrm{kDa}$; G1), 19 (glycates made from DX that had an $M_{W}$ value of $3.5 \mathrm{kDa}$; G3.5), 24 (glycates made from DX that had an $M_{W}$ value of $10 \mathrm{kDa}$; G10), 36 (glycates made from DX that had an $M_{W}$ value of 150 $\mathrm{kDa}$; G150), or $48 \mathrm{~h}$ (glycates made from DX that had $M_{W}$ values of 500 and 2,000 kDa; G500 and G2000, respectively). Incubation times were varied to try to achieve similar levels of glycates after the heating step. The optimal incubation time for glycation was selected to minimize the formation of undesirable color and offflavors resulting from the advanced stage of the Maillard reaction. Temperatures $>65^{\circ} \mathrm{C}$ increased the risk of unwanted whey protein denaturation or aggregation. It was also unclear how unreacted protein or advanced Maillard products would affect the IgE-binding test; thus, we wanted to limit the concentration of these components. The concentration of DX in all cases was very high $(30 \%)$, such that macromolecular crowding inhibited denaturation and aggregation of whey proteins during this type of glycation reaction (Zhu et al., 2008). At higher DX concentrations (>30\%), DX molecules tended to lose solubility during the glycation reaction. Heating time was optimized based on the approach of Zhu et al. (2008) - that is, monitoring Schiff base formation and yellow-brown color changes (due to formation of advanced Maillard reaction products) by measuring difference UV absorbance at $305 \mathrm{~nm}$ (Zhu et al., 2008) and $420 \mathrm{~nm}$ (Jiménez-Castaño et al., 2007), respectively. The reaction was stopped by cooling the solution in ice water.

\section{Purification of WPI-DX Glycates via Ion Exchange Chromatography}

Crude glycate mixtures were diluted 5-fold with 10 $\mathrm{m} M$ SPB (pH 6.5) and adjusted to $\mathrm{pH} 5.0$ with 0.5 $M \mathrm{HCl}$. Any precipitated, unglycated protein (UP) was removed by centrifugation at $5,200 \times g$ for 40 min (Bund et al., 2012). The supernatant was filtered through a $0.7-\mu \mathrm{m}$ filter, and $\mathrm{pH}$ was adjusted to 2 or 3 with $1 M \mathrm{HCl}$ based on different purification conditions that yielded maximum WPI-DX glycate purity (based on preliminary experiments). Feed solution was loaded onto a 100-mL cation exchange column (MacroCap SP cation exchange resin; GE Healthcare, Uppsala, Sweden). Glycation of WPI with DX blocks some amino groups of the protein, leading to WPI-DX glycates that were less charged than the unreacted WPI. The UV detector detects 3 peaks during elution of glycates using the salt gradient. Dextran is uncharged, does not bind to the column, and was eluted by the running buffer. The low salt peak contained primarily glycated protein with little or no UP, and the high salt peak contained primarily UP with little or no glycated protein (Bund et al., 2012).

The purification conditions for different glycates were performed at different $\mathrm{pH}$ values and $\mathrm{NaCl}$ levels to yield maximum purity of glycates. The unreacted DX was removed by washing the column with equilibration buffer $(25 \mathrm{~m} M$ phosphatidic acid buffer at $\mathrm{pH} 2$ or 50 $\mathrm{m} M$ lactic acid buffer at $\mathrm{pH} 3$ ). The WPI-DX glycates were eluted by a gradient of 0 to $0.55 \mathrm{M} \mathrm{NaCl}$, with absorbance monitored at $305 \mathrm{~nm}$ by UV detector (Optical Unit UV-1; GE Healthcare). Elution material was desalted by an ultrafiltration membrane ( $M_{W}$ cut-off of $10 \mathrm{kDa}$; Millipore Corp.) and dialyzed against Milli-Q water using dialysis membrane with an $M_{W}$ cut-off of 6,000 to 8,000 Da. The purified glycates were lyophilized and stored at $-20^{\circ} \mathrm{C}$.

\section{SDS-PAGE Analysis to Assess Extent of Glycation}

Sodium dodecyl sulfate-PAGE was performed on native WPI and WPI-DX glycates to determine the extent of glycation of WPI (Zhu et al., 2008) and to compare the size distributions for WPI glycated with DX of different $M_{W}$. The SDS-PAGE analyses were carried out on a Mini-Protean 3 cell (BioRad Laboratories, Hercules, CA) based on the method of Laemmli (1970). The samples were diluted with Laemmli sample buf- 
fer and loaded onto 10-well Mini-Protean TGX precast gel (Tris-HCl, 4-20\%). Loading concentration of each sample was $3 \mathrm{mg} / \mathrm{mL}$ on a protein basis. Gels were run for $35 \mathrm{~min}$ at $200 \mathrm{~V}$ in a $25 M$ Tris- $\mathrm{HCl}$ buffer $(\mathrm{pH}$ 8.3, including $0.192 M$ glycine and $0.1 \%$ wt/wt SDS) at room temperature. Afterward, gels were stained for protein with Coomassie Brilliant Blue G-250 (Bio-Rad Laboratories) or for glycoproteins using a GelCode glycoprotein staining kit (Pierce Biotechnology, Rockford, IL).

\section{SEC-MALLS}

We performed SEC-MALLS according to the method of Wang and Lucey (2003). Lyophilized WPI, DX (1, $3.5,10,150,500$, and 2,000 kDa), and glycates (G1, G3.5, G10, G150, G500, and G2000) were reconstituted in $20 \mathrm{mM}$ imidazole and $50 \mathrm{mM} \mathrm{NaCl}$ buffer $(\mathrm{pH} \mathrm{7})$ and left overnight at $5^{\circ} \mathrm{C}$ for hydration. Sample concentrations were $0.2 \%$ (wt/wt) for WPI, $1 \%$ (wt/wt) for DX, and $0.4 \%$ (wt/wt) for glycates to give a high but not oversaturated refractive index signal. Prior to loading, samples were ultracentrifuged for $90 \mathrm{~min}$ at $52,000 \times$ $g$ and filtered through a $0.22-\mu \mathrm{m}$ filter to remove large particles or contaminants. A total of $50 \mu \mathrm{L}$ of each sample was then loaded onto the SEC system at a flow rate of $0.5 \mathrm{~mL} / \mathrm{min}$. The $\mathrm{SEC}$ system consisted of a Waters 600 HPLC unit (Waters Corp., Milford, MA) connected to an SEC Superose 6HR 10/30 and a SEC Superose 12HR 10/30 column (Amersham Pharmacia Biotech AB, Uppsala, Sweden). The SEC columns were maintained at approximately $40^{\circ} \mathrm{C}$ throughout the experiments. Three detectors were used for data collection: a UV detector operating at $280 \mathrm{~nm}$ (model 996; Waters Corp.), a DAWN-EOS MALLS photometer system (Wyatt Technology, Santa Barbara, CA) fitted with a helium neon laser $(\lambda=690 \mathrm{~nm})$, and a K-5 flow cell and a differential refractive index detector (model 2410; Waters Corp.). The $M_{W}$ distributions were calculated by ASTRA software (version 4.73.04; Wyatt Technology, Santa Barbara, CA) using the Debye fit method (Lucey et al., 2000). The Dn/dc values (refractive index increment) used were $0.185,0.140$, and $0.160 \mathrm{~mL} / \mathrm{g}$ for WPI, DX, and glycates (G1, G3.5, G10, G150, G500, and G2000), respectively.

\section{Compositional Analysis of Glycates}

The bicinchoninic acid assay was used to estimate the total protein content and was verified by the Kjeldahl method. Purified glycates were dissolved in 10 $\mathrm{m} M$ SPB. The total protein content was assayed using a bicinchoninic acid assay kit (Pierce Biotechnology). Protein contents were calculated using a WPI standard curve. The carbohydrate content was determined using the phenol sulfuric acid assay by DuBois et al. (1956). Briefly, $2 \mathrm{~mL}$ of sample solution $(0.1 \mathrm{mg} / \mathrm{mL}$, carbohydrate based) was mixed with $5 \%$ phenol and $5 \mathrm{~mL}$ of sulfuric acid and placed in a water bath at $30^{\circ} \mathrm{C}$ for 15 min. Afterward, the optical density was determined at $490 \mathrm{~nm}$ (Spectramax Plus; Molecular Devices, Sunnyvale, CA). The carbohydrate content was calculated using a DX standard curve. Molar binding ratios were determined as described by Hattori (2002).

The molar concentration of accessible $\mathrm{NH}_{2}$ groups in the glycates was measured according to Nielsen et al. (2001) and Church et al. (1983). Briefly, lyophilized glycates and WPI were reconstituted in Milli-Q water to a final protein concentration of $1 \mathrm{mg} / \mathrm{mL}$. A total of $400 \mu \mathrm{L}$ of each sample was mixed with $3 \mathrm{~mL}$ of an $O$-phtaldialdehyde solution containing $1 \mathrm{mg} / \mathrm{mL}$ of SDS and $0.88 \mathrm{mg} / \mathrm{mL}$ of dithiothreitol. After $2 \mathrm{~min}$, the optical density was measured at $340 \mathrm{~nm}$. The concentration of free $\mathrm{NH}_{2}$ groups (nmol/L) was then calculated using a serine standard curve.

\section{AFM of Glycates}

The AFM images of the glycates were measured according to Ikeda and Morris (2002) and Liu and Zhong (2015). All AFM experiments were performed using a BioScope Catalyst atomic force microscope equipped with a Nanoscope IIIa controller (Bruker, Santa Barbara, CA). Purified WPI-DX glycates were dissolved in an SPB buffer solution to give concentrations of 0.5 to $1 \mu \mathrm{g} / \mathrm{mL}$. A $2-\mu \mathrm{L}$ volume of the solution was drop deposited on a freshly cleaved mica surface and evenly spread to minimize intermolecular aggregation, enabling us to image individual molecules after they were air dried. Atomic force microscopy imaging was performed under peak force tapping mode using silicon nitride AFM tips (ScanAsyst-air; Bruker, Camarillo, CA) with a nominal spring constant of $0.4 \mathrm{~N} / \mathrm{m}$ and a resonant frequency of approximately $70 \mathrm{kHz}$. At least 5 images were obtained per sample. Obtained images were analyzed using the software NanoScope Analysis (Bruker, Santa Barbara, CA).

\section{IgE-Binding Capacity of WPI-DX Glycates}

A total of $200 \mu \mathrm{L}$ of composite blood sera and $20 \mu \mathrm{L}$ of heated WPI or glycates was mixed to $50 \mu \mathrm{g} / \mathrm{mL}$ on a protein basis based on the method of Sampson (2001). Samples were incubated at $5{ }^{\circ} \mathrm{C}$ overnight and loaded onto an ImmunoCAP 100 system (Phadia); WPI caps were used as a stationary phase (ImmunoCAP allergen Rf236; Phadia). Control experiments with $10 \mathrm{mM}$ SPB 
and DX10 were also undertaken. The IgE levels measured after preincubation with an SPB control $(0 \mu \mathrm{g} /$ $\mathrm{mL}$ of protein) were regarded as the maximum WPIspecific IgE response (100\%). All other results were expressed as a percentage thereof.

\section{RESULTS}

\section{Optimizing the Glycation and Purification of WPI-DX Glycates}

The WPI was glycated with DX of different $M_{W}$ by the aqueous heating method (Zhu et al., 2008, 2010), and the reaction was stopped to avoid the Maillard reaction moving to the advanced stage (i.e., forming brown colors, known as melanoidins; de Oliveira et al., 2016). Glycation conditions were optimized to provide a high concentration of WPI-DX glycates for the IgE-binding test, with a low level of brown color development $(<10 \%$ increase in absorbance measured by difference UV at $420 \mathrm{~nm}$ ). The optimal incubation conditions selected for the DX with different $M_{W}$ were $62^{\circ} \mathrm{C}$ for $7 \mathrm{~h}$ (G1), $19 \mathrm{~h}$ (G3.5), $24 \mathrm{~h}$ (G10), and 36 $\mathrm{h}$ (G150) and $64^{\circ} \mathrm{C}$ for $48 \mathrm{~h}$ (G500 and G2000). The optimum purification condition to elute glycates from the ion exchange column was $0.53 \mathrm{M} \mathrm{NaCl}$ at $\mathrm{pH} 2$ to reach a purity $>95 \%$.

The SDS-PAGE electrophoretograms of glycates stained with a protein stain (Coomassie Blue) and a carbohydrate stain (periodic acid stain) confirmed the formation of stable covalently bound glycates (Figure 1). By comparing the raw materials and solutions after heating for $24 \mathrm{~h}$, faint magenta bands appeared after heating, demonstrating the presence of glycates (i.e., pure DX does not migrate through the gel electric field because it is uncharged). Lane 4 was for glycates eluted by $0.53 \mathrm{M} \mathrm{NaCl}$ from the ion exchange column; the intense, smeared band stained positive for protein and carbohydrate, which indicated covalent bond formation and a broad size distribution for the glycates formed. Unreacted whey protein, primarily containing $\beta$-LG, was eluted by $1 M \mathrm{NaCl}$ (lane 5). Under nonreducing conditions, BSA and $\alpha$-LA were aggregated after heat treatment (De Wit, 1990). These UP were very likely removed after $\mathrm{pH}$ adjustment and centrifugation (data not shown). After purification, only low levels of unreacted $\beta-\mathrm{LG}$ remained, which yielded glycates with a high purity (lane 4).
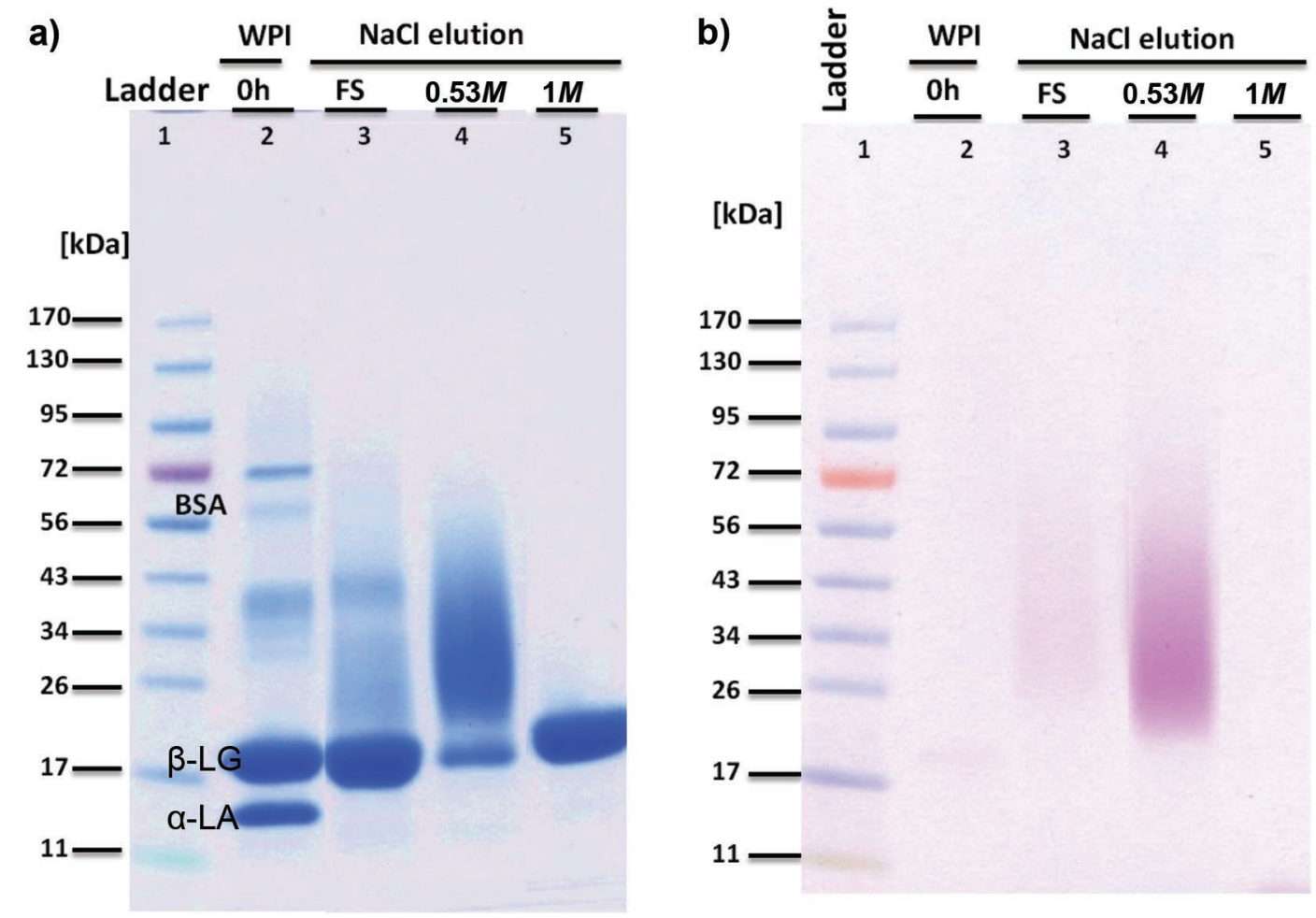

Figure 1. Sodium dodecyl sulfate-PAGE overview of the production and purification of glycates made from dextran (DX) that had a molar mass $\left(M_{W}\right)$ value of $3.5 \mathrm{kDa}$ in Laemmli buffer with $5 \%$ of 2-mercaptoethanol. (a) Protein-specific Coomassie Brilliant Blue staining. (b) Glycoprotein-specific periodic acid staining. Lane 1: $M_{W}$ standard; lane 2: whey protein isolate (WPI) without treatment; lane 3: feed conjugate solution (FS; mixture of WPI and DX after $19 \mathrm{~h}$ of heat treatment); lane 4: peak fraction eluted by $0.53 \mathrm{M}$ (mol/L) NaCl from cation exchange column; lane 5: peak fraction eluted by $1 \mathrm{M} \mathrm{NaCl}$ from cation exchange column. Color version available online. 
Figure 2 shows a reducing SDS-PAGE gel of WPI glycated with DX with different $M_{W}$ from 1 to 2,000 $\mathrm{kDa}$. Bands with $M_{W}$ of 14,18 , and $66 \mathrm{kDa}$ in lane 1 correspond to $\alpha-\mathrm{LA}, \beta-\mathrm{LG}$, and BSA, respectively. The G1 glycate had 1 intense band with apparent $M_{W}$ range of 20 to $40 \mathrm{kDa}$. The smeared bands in the following lanes (from lanes 3 to 7 ) shifted to a higher $M_{W}$ range (from 20 to approximately $72 \mathrm{kDa}$ in G3.5 to 20 to approximately $>170 \mathrm{kDa}$ in G2000), indicating larger $M_{W}$ for these glycates. The increase of $M_{W}$ could be attributed to the change in the binding ratios of WPI and DX, which are discussed later. The intense bands in lanes 5, 6, and 7, corresponding to $M_{W}$ values $>200$ $\mathrm{kDa}$, indicated the presence of glycates that were too large to migrate through the stacking gel. The intensity of the magenta bands increased from lanes 2 to 7 , confirming the higher carbohydrate content in G500 and G2000 compared with smaller $M_{W}$ WPI-DX glycates because the intensity of the color of the magenta bands is proportional to the carbohydrate content (Dubray and Bezard, 1982).

\section{Characterization of Glycates}

Results for the protein and carbohydrate contents of the glycates are summarized in Table 1. The G500 and G2000 glycates had a carbohydrate content of $>80 \%$ due to their large $M_{W}$ limiting the binding of WPI, whereas using smaller DX (i.e., $1 \mathrm{kDa}$ ) resulted in purified glycates with very high protein level. These trends were in agreement with previous reports (Hattori, 2002; Liu et al., 2012). The $M_{W}$ values of WPI, DX3.5, and the WPI-DX glycate G3.5 were determined by SECMALLS (Figure 3), which indicated an increased $M_{W}$ for WPI after glycation with DX3.5. Figure 4 shows the refractive index signal of DX and its corresponding glycates. With the increase in the $M_{W}$ of DX, the $M_{W}$ values of the corresponding glycates also increased (Figure $4 \mathrm{~b}$ ), consistent with the results of SDS-PAGE (Figure 2). The refractive index signal for purified glycates (Figure 4b) was consistent with the result from UV signal at $280 \mathrm{~nm}$, which indicated no interference signal from unreacted DX.

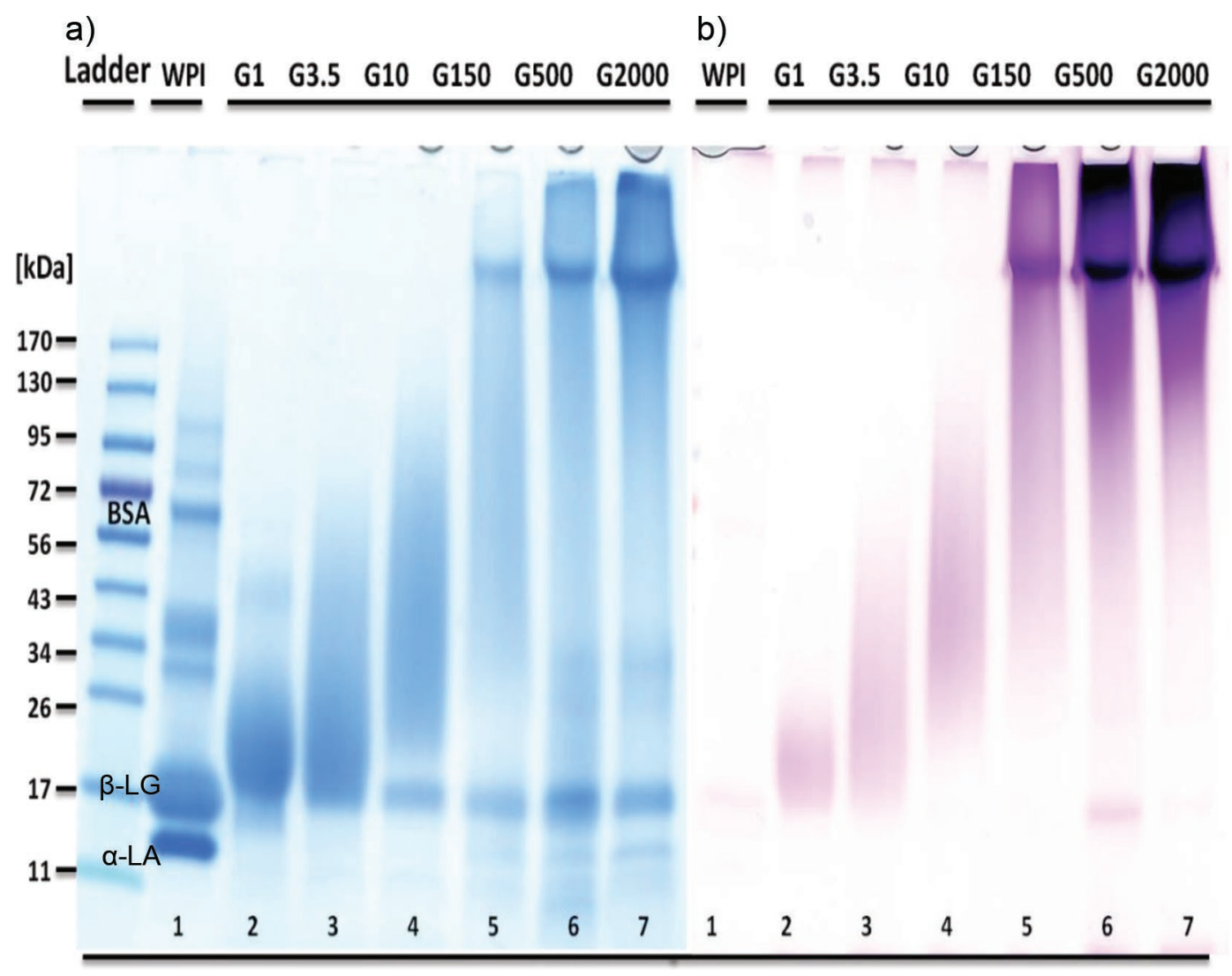

Figure 2. Sodium dodecyl sulfate-PAGE gels of purified glycates made from dextrans that had molar mass values of 1 (G1), 3.5 (G3.5), 10 (G10), 150 (G150), 500 (G500), and 2,000 (G2000) kDa in Laemmli buffer and 5\% of 2-mercaptoethanol. (a) Protein-specific Coomassie Brilliant Blue staining. (b) Glycoprotein-specific periodic acid staining. WPI $=$ whey protein isolate. Color version available online. 


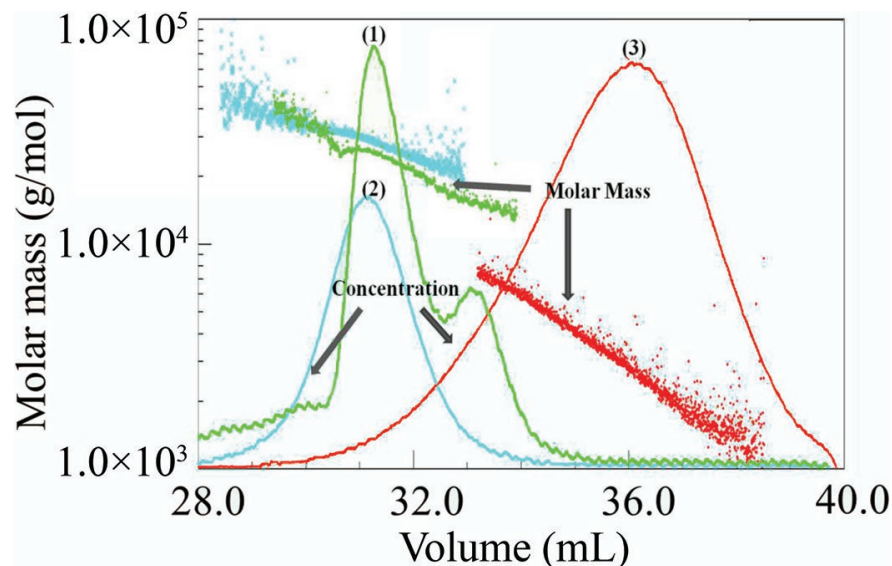

Figure 3. Size-exclusion chromatography-multiangle laser light scattering elution profile and molar mass distribution of whey protein isolate (1, green), glycates made from dextran that had a molar mass value of $3.5 \mathrm{kDa}(2$, blue), and dextran with a molar mass of $3.5 \mathrm{kDa}$ $(3$, red $)$ as a function of elution volume.

The estimated $M_{W}$ of WPI was $27.6 \mathrm{kDa}$ (Figure 3 ), and the estimated $M_{W}$ values of series DX were almost equivalent to the labeled $M_{W}$ values from the companies, which were used to calculate the binding ratios between WPI and DX. Taking G1 and G10 as examples, the $M_{W}$ of DX1 and DX10 were 1.1 and $8.7 \mathrm{kDa}$, respectively. According to the results of protein and carbohydrate contents as well as the estimated $M_{W}$ for WPI and DX, molecular binding ratios between WPI and DX were 1:3.5 for G1 and 1:2.5 for G10 (Table 1). This indicated that in G1, each protein molecule was attached by 3 to 4 DX molecules on average, whereas G10 had 2 to 3 DX bound per protein molecule. However, for the larger glycates (peaks 4,5 , and 6 ) with very large range of $M_{W}$, the average $M_{W}$ is not very accurate due to the polydispersity (Figure 4). Lower binding of DX to WPI occurred as the $M_{W}$ of DX increased (Table 1), as was expected due to steric difficulties in gaining surface (a)

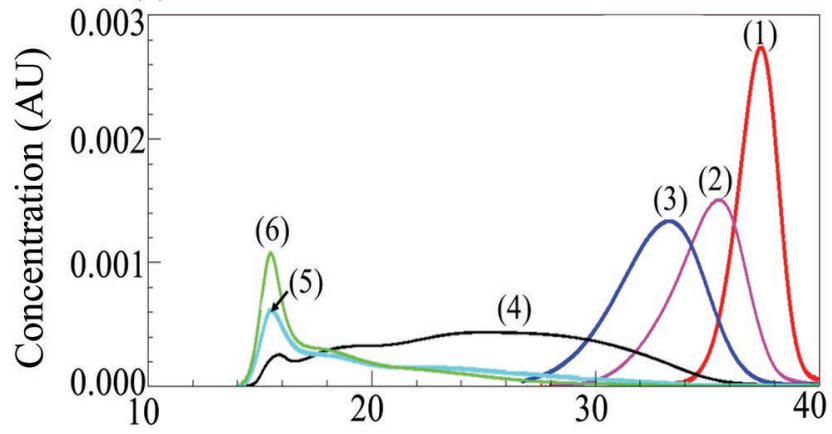

(b)

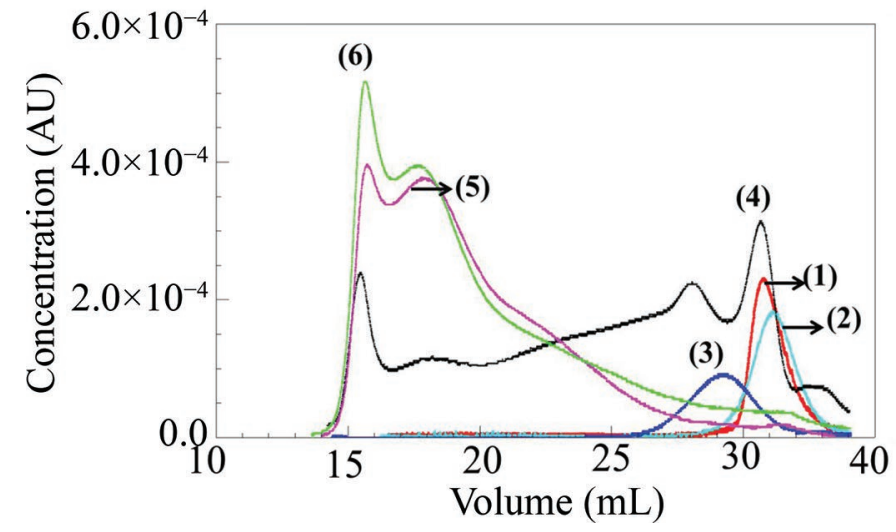

Figure 4. Size-exclusion chromatography-multiangle laser light scattering elution profile of dextran (DX) and its corresponding glycates. (a) Refractive index signal of eluted DX with a molar mass $\left(M_{W}\right)$ of $1 \mathrm{kDa}(1$, red), $3.5 \mathrm{kDa}(2$, pink), $10 \mathrm{kDa}(3$, navy), $150 \mathrm{kDa}$ (4, black), $500 \mathrm{kDa}(5$, blue), and $2.000 \mathrm{kDa}(6$, green) as a function of elution volume. (b) Refractive index signal of eluted glycates made from DX that had $M_{W}$ values of $1 \mathrm{kDa}$ (1, red), $3.5 \mathrm{kDa}(2$, blue), 10 $\mathrm{kDa}(3$, navy), $150 \mathrm{kDa}$ (4, black), $500 \mathrm{kDa}$ (5, pink), and 2,000 kDa (6, green).

Table 1. Summary of characterization of purified glycates, including protein content, carbohydrate content (dry weight basis), and average molar mass $\left(M_{W}\right)^{1}$

\begin{tabular}{lccccc}
\hline Sample $^{2}$ & $\begin{array}{c}\text { Protein } \\
(\%)\end{array}$ & $\begin{array}{c}\text { Carbohydrate } \\
(\%)\end{array}$ & $\begin{array}{c}M_{W} \\
(\mathrm{kDa})\end{array}$ & $\begin{array}{c}\text { Polydispersity index } \\
\left(M_{W} / M_{n}^{3}\right)\end{array}$ & $\begin{array}{c}\text { WPI:DX molar } \\
\text { binding ratio }\end{array}$ \\
\hline WPI & $92.8 \pm 0.9^{\mathrm{a}}$ & $0.5 \pm 0.1^{\mathrm{g}}$ & 27.2 & $1.15 \pm 0.13$ & - \\
G1 & $80.5 \pm 2.7^{\mathrm{b}}$ & $11.2 \pm 0.2^{\mathrm{f}}$ & 31.2 & $1.04 \pm 0.12$ & $1: 3-4^{\mathrm{a}}$ \\
G3.5 & $67.9 \pm 1.7^{\mathrm{c}}$ & $18.1 \pm 0.9^{\mathrm{e}}$ & 34.3 & $1.02 \pm 0.21$ & $1: 2-3^{\mathrm{b}}$ \\
G10 & $54.5 \pm 2.1^{\mathrm{d}}$ & $36.9 \pm 1.3^{\mathrm{d}}$ & 40.2 & $1.03 \pm 0.20$ & $1: 2-3^{\mathrm{b}}$ \\
G150 & $25.1 \pm 0.9^{\mathrm{e}}$ & $63.2 \pm 1.0^{\mathrm{c}}$ & 249.5 & $7.73 \pm 0.54$ & $1: 1-2^{\mathrm{c}}$ \\
G500 & $15.8 \pm 1.0^{\mathrm{f}}$ & $79.7 \pm 0.6^{\mathrm{b}}$ & 427.6 & $2.23 \pm 0.40$ & $1: 1^{\mathrm{d}}$ \\
G2000 & $14.7 \pm 0.8^{\mathrm{g}}$ & $81.4 \pm 1.3^{\mathrm{a}}$ & 450.8 & $3.61 \pm 0.56$ & $1: 1^{\mathrm{d}}$ \\
\hline
\end{tabular}

${ }^{\mathrm{a}-\mathrm{g}}$ Means within a row with different superscripts differ $(P<0.05)$.

${ }^{1}$ Data are means of triplicate trials, presented as means $\pm \mathrm{SD}$.

${ }^{2} \mathrm{WPI}=$ whey protein isolate; G1-G2000 = glycates made from dextrans (DX) that had $M_{W}$ values of 1 (G1), 3.5 (G3.5), 10 (G10), 150 (G150), 500 (G500), and 2,000 (G2000) kDa.

${ }^{3} M_{n}=$ number average molar mass. 


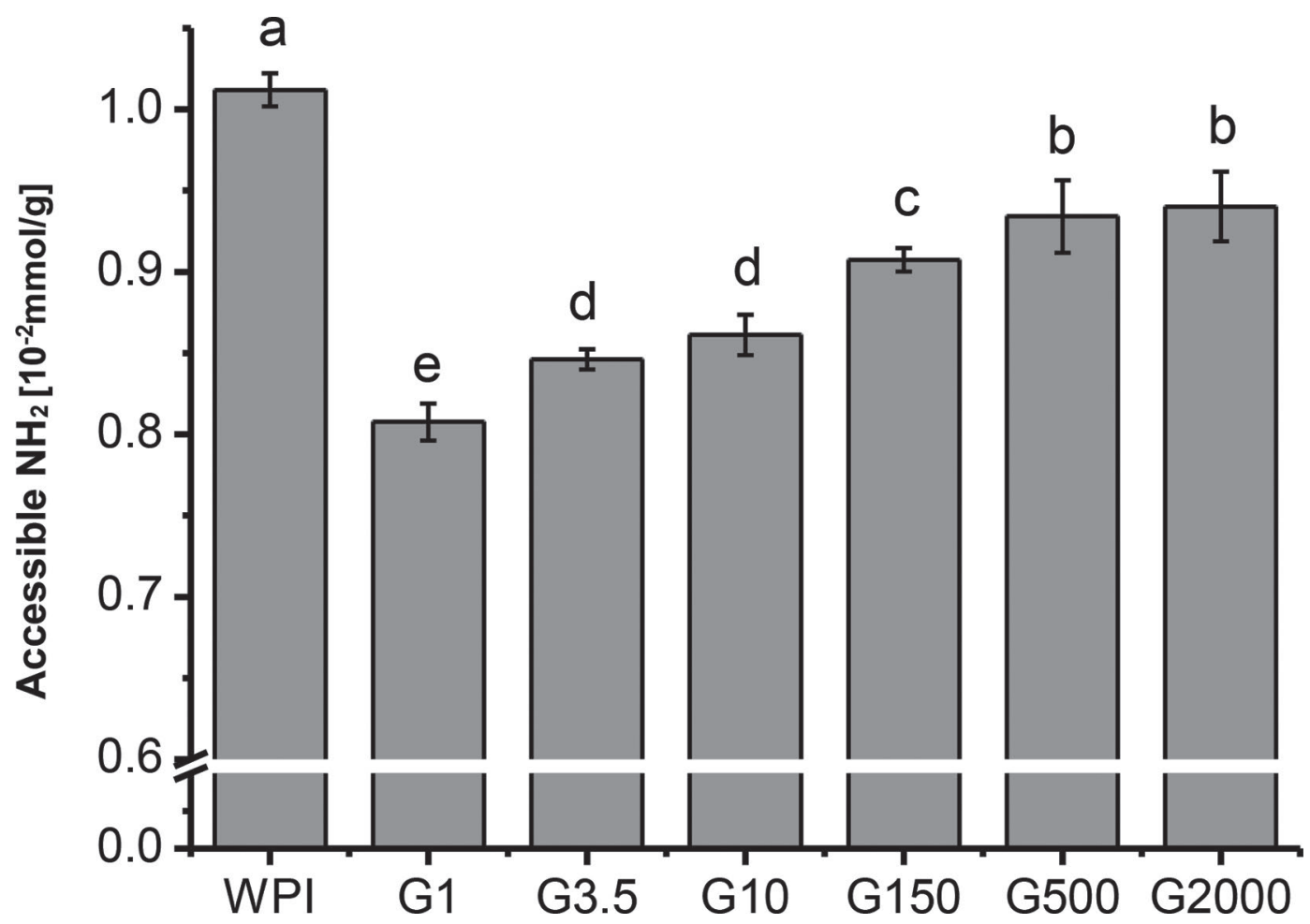

Figure 5. Accessible $\mathrm{NH}_{2}$ groups in native whey protein isolate (WPI) and WPI-dextran glycates determined via $O$-phthaldialdehyde method. G1-G2000 = glycates made from dextrans that had molar mass values of 1 (G1), 3.5 (G3.5), 10 (G10), 150 (G150), 500 (G500), and 2,000 (G2000) kDa. Data are presented as means \pm SD. Means with different letters (a-e) differ $(P<0.05)$ using a $t$-test.

accessibility with the presence of a bulky DX already bound to the WPI molecule.

The WPI to DX binding ratios were further investigated by measuring the levels of accessible $\mathrm{NH}_{2}$ groups in the final glycates, expressed on a constant protein concentration basis (Figure 5). After glycation, the amount of accessible $\mathrm{NH}_{2}$ groups in the protein moiety was reduced by up to $20 \%$ in G1 and 6 to $7 \%$ in G2000 and G500, respectively, which was consistent with the lower binding ratios. Incubation of WPI under the same conditions used for glycation did not lead to a detectable loss of $\mathrm{NH}_{2}$ groups (data not shown).

The AFM images (Figure 6) were captured to investigate the morphology of DX, WPI, and WPI-DX glycates. Figure $6 \mathrm{a}$ and $\mathrm{b}$ shows the topographical images of individual DX10 and WPI molecules. Samples were analyzed at $1 \mu \mathrm{g} / \mathrm{mL}, \mathrm{pH} 6.5$ solution, and spread onto mica with typical average diameters of 10 to 20 $\mathrm{nm}$ for DX and 20 to $30 \mathrm{~nm}$ for WPI, which were in agreement with previous studies (Ikeda, 2003). Dextran is a branched (1-6 linked) polysaccharide that has a flexible and extended polymer structure. In the AFM images, DX had largely a club-shaped feature as the random coil collapsed on the mica surface (Figure $6 a)$. Figure $6 \mathrm{~b}$ confirmed that the whey protein had a typical globular structure, also showing a higher brightness than the DX, indicating a larger value for particle height. Figure 6c and d shows images for G10 and G150. In Figure 6c, the DX10 cluster appears to be bound to the surface of the WPI protein instead of wrapping around the protein molecule. In G150 (Figure $6 \mathrm{~d}$ ), the DX appeared to be extended into the solution, whereas more of the protein surface visually appeared to be covered by DX in G10 (Figure 6c).

\section{IgE-Binding Capacity of WPI-DX Glycates}

The IgE-binding capacity of the purified glycates was monitored after incubation with sera from blood samples obtained from patients with CMPA, followed by the IgE-specific ImmunoCAP test. The sera from 8 CMPA patients were combined equally to avoid individual differences. There was no statistical difference between negative control (SPB solution), wheat protein, and DX, indicating that no nonspecific IgE binding had occurred during preincubation (data not shown). After preincubation, the levels of WPI-specific IgE detected by the ImmunoCAP test were higher for sera incubated with glycates compared with native WPI. The actual binding capacity was calculated by subtracting the 

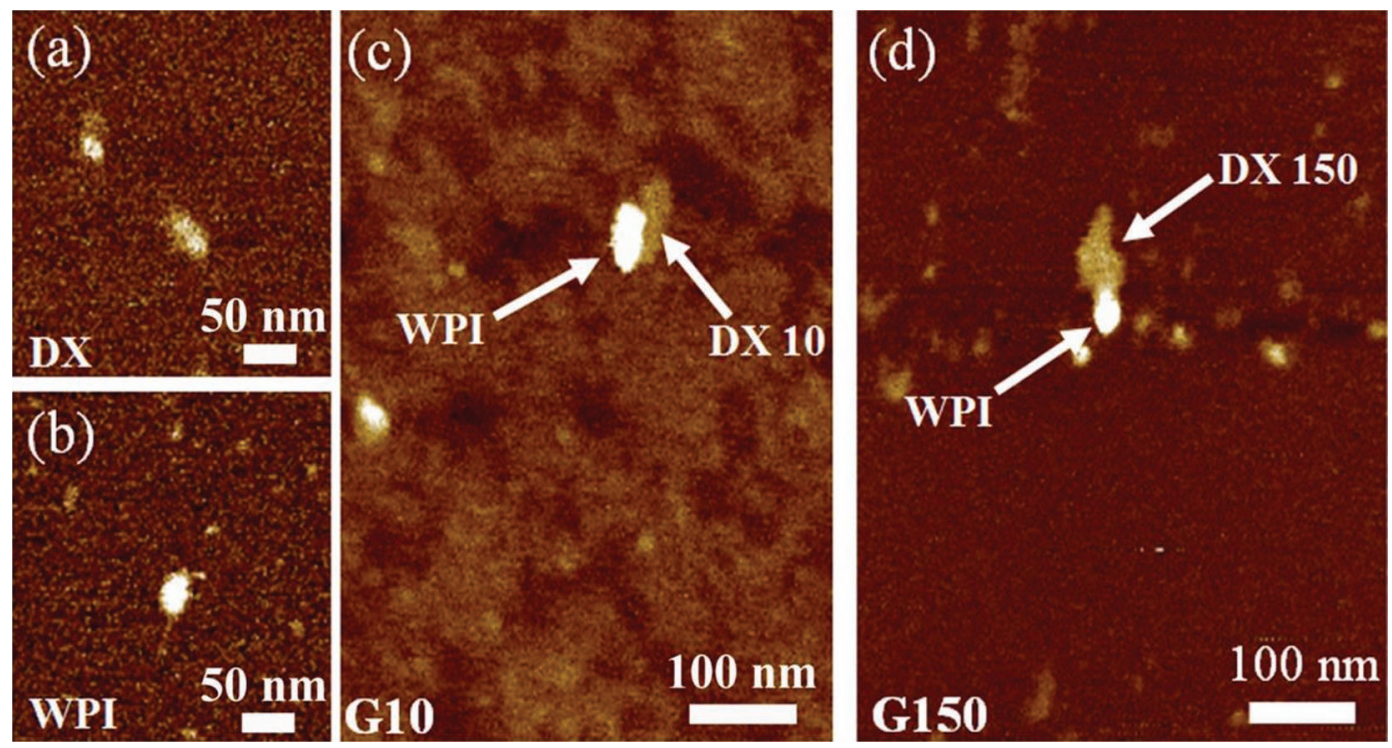

Figure 6. Atomic force microscopy topographical images of individual molecules of (a) dextran (DX) with a molar mass of $10 \mathrm{kDa}$, (b) whey protein isolate (WPI), (c) purified glycates made from DX that had a molar mass value of $10 \mathrm{kDa}$ (G10), and (d) glycates made from DX that had a molar mass value of $150 \mathrm{kDa}$ (G150) dissolved in sodium phosphate buffer solution at $\mathrm{pH} 6.5$ and a concentration of 0.5 to $1 \mu \mathrm{g} / \mathrm{mL}$. Color version available online.

detected signal from the control group, which was sera incubated with SPB buffer. The WPI-DX glycates had a significantly lower WPI-specific IgE-binding capacity than nonglycated WPI (Figure 7). Glycates with DX of small $M_{W}$ (G1, G3.5, and G10) had a much lower

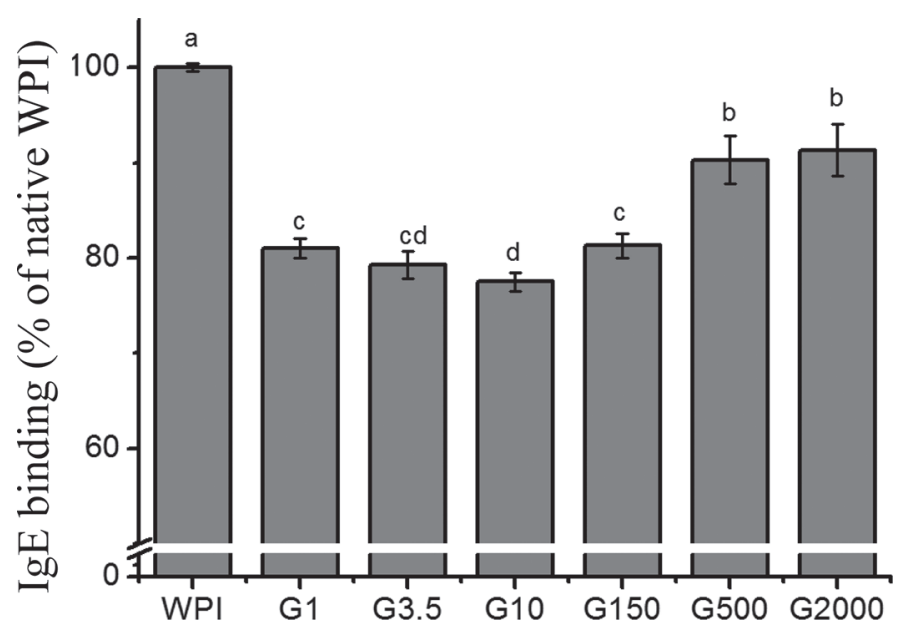

Figure 7. Immunoglobulin E-binding capacity $(\mathrm{kUA} / \mathrm{L})$ as determined by ImmunoCAP (Phadia, Uppsala, Sweden) test of whey protein isolate (WPI) and glycates made from dextrans that had molar mass values of $1 \mathrm{kDa}$ (G1), $3.5 \mathrm{kDa}$ (G3.5), $10 \mathrm{kDa}$ (G10), $150 \mathrm{kDa}$ (G150), $500 \mathrm{kDa}$ (G500), and 2,000 kDa (G2000). Results are expressed as amount of $\mathrm{IgE}$ bound to the native WPI $(10 \mathrm{kUA} / \mathrm{L})$ or glycates. The results are given as the means of triplicate measurements, presented as means $\pm \mathrm{SD}$. Means with different letters $(\mathrm{a}-\mathrm{d})$ differ $(P$ $<0.05)$ using a $t$-test.
WPI-specific IgE-binding capacity than large glycates (i.e., G500, G2000), with the lowest IgE-binding capacity obtained with G10 glycates.

\section{DISCUSSION}

Our study aimed to explore whether whey protein glycates reduced $\operatorname{IgE}$ binding and what size glycate would give a greater reduction in $\operatorname{IgE}$ binding. The size of the polysaccharide used for glycation has been reported to be an important factor affecting some functional properties for glycates. In our study, glycates made with $10-\mathrm{kDa}$ DX resulted in the lowest $\mathrm{IgE}$ binding capacity (Figure 7). The effect of the $M_{W}$ of the polysaccharide on the functionality of whey protein glycates has been previously studied, but conflicting results have been reported for the optimum size. Several studies have been conducted on the stability of emulsions prepared with different sizes of glycates. Akhtar and Dickinson (2007) reported that WPI with several bound small maltodextrin molecules could effectively act as an emulsion stabilizer. Dickinson (2008) suggested that glycation with a polysaccharide of 5 to $10 \mathrm{kDa}$ should be sufficient to help improve emulsion stability. Wooster and Augustin (2006) indicated that glycation of whey protein with any DX, irrespective of the $M_{W}$ of DX, improved emulsion stability, whereas Dunlap and Côté (2005) indicated that increasing the $M_{W}$ of DX up to $150 \mathrm{kDa}$ increased that stability of emulsions prepared with whey protein glycates. 
With the use of smaller $M_{W} \mathrm{DX}$, the glycates seemed more compact compared with glycates made with DX of higher $M_{W}$, where structures extending out from the globular protein aggregates were observed (Figure 6). The highly flexible DX molecule probably contributed to its extended appearance in glycates. Only a few studies have been conducted on the structure of glycates (Liu et al., 2012). Xu and Yao (2009) glycated DX with acid-soluble soy protein by the dry heating method, and glycates with extended structures ("tails") could be observed with AFM (probably due to the attachment of flexible DX molecules). Similar types of images were observed for our larger $M_{W}$ WPI-DX glycates (Figure 6d). Yadav et al. (2012) used scanning electron microscopy to study the microstructure of corn fiber gum that was glycated with whey protein. They indicated that whey proteins became attached to the ends of stiff extended corn fiber gum molecules. Liu and Zhong (2015) observed nanometer-sized particles that were in a similar size range to our WPI-DX glycates but smaller after glycation (by the dry heating method) of WPI with lactose and maltodextrin (which are smaller $M_{W}$ carbohydrates).

The in vitro allergenicity of glycates was evaluated by measuring IgE-binding capacity of the purified glycates via the IgE-specific ImmunoCAP test (Figure 7). In this study, purified WPI-DX glycates were incubated with the blood sera from actual patients with CMPA. All the samples were incubated with the same amount of protein. Thus, we reported for the first time the effect of glycation assessed in the actual system in which whey proteins provoke the negative immune response associated with CMPA. No nonspecific IgE binding occurred during preincubation, as was also demonstrated by Böttger (2013). The ImmunoCAP test provided a more direct and accurate estimation of IgE-binding capacity of WPI-DX glycates than ELISA or other immunoassays performed with animal sera, as performed in some previous studies. The IgE-binding capacity results (Figure 7) demonstrated that glycation of WPI with DX resulted in a significant reduction in the allergenicity of whey protein. A maximum reduction of about $20 \%$ in IgE binding was achieved with G10 compared with unglycated WPI (Figure 7).

The reduction in the $\operatorname{IgE}$ binding was likely due to the shielding effect created by the attachment of the flexible linear chains of the DX on the linear epitopes of the protein. This steric hindrance (or physical barrier) around the protein surface could limit the accessibility of $\operatorname{IgE}$ to bind to the protein allergy sites (Hattori et al., 2004; Taheri-Kafrani et al., 2009; Corzo-Martínez et al., 2010). This IgE-binding reduction could be affected by the $M_{W}$ of DX as well as the number of
DX bound to the proteins. Compared with glycation of WPI with large $M_{W}$ DX (G500 and G2000), glycation of WPI with small $M_{W}$ DX (G1, G3.5, and G10) resulted in a higher binding ratio between WPI and DX (up to 4 DX molecules bound per protein molecule; Table 1).The high binding ratio achieved with low $M_{W}$ DX seemed to result in greater surface coverage visually on whey proteins compared with large $M_{W}$ DX. It is evident from the AFM images that after glycation the $10-\mathrm{kDa}$ DX covered a substantial area on the surface of whey proteins (Figure 6c), potentially reducing the accessible surface for IgE binding. In comparison, the larger $150-\mathrm{kDa}$ DX covered a much smaller surface area, with most of the flexible DX molecule extending out into the solvent due to the random coil feature of DX (Figure 6d).

Allergenic epitopes are of both conformational and linear nature and are mostly randomly distributed throughout the protein molecule. For $\beta$-LG, the most dominant epitopes were found to be within peptides $f(41-60), f(102-124)$, and $f(149-162)$. They are recognized by $>90 \%$ of CMPA patients, and each one accounts for about 10 to $15 \%$ of the total $\beta$-LG allergenicity (Wal, 2002). Up to 7 specific IgE-binding epitopes have been reported for $\beta-\mathrm{LG}$, which also has several potential conformational epitopes (Järvinen et al., 2001; Wal, 2002; Niemi et al., 2007). Immunoglobulin E binding to both linear and conformational epitopes can trigger an allergic reaction. Glycation of WPI with DX may also introduce structural alterations to WPI, leading to a loss of accessibility to these epitopes due to this conformational change. Liu et al. (2016) reported that during an extensive dry-heating glycation process $\left(130^{\circ} \mathrm{C}\right.$ for $\left.20 \mathrm{~min}\right)$, most of the Maillard reaction sites were located in the reported conformational epitope areas on whey proteins. They observed that structural changes during this heating process contributed to the decreased IgE-binding capacity (Liu et al., 2016). Significant structural changes have been reported to be caused by the dry heating processes commonly used for glycation (Liu et al., 2012). Zhu et al. (2008) reported that protein unfolding occurs during the aqueous glycation reaction of WPI with DX, although the temperatures used were much lower $\left(<65^{\circ} \mathrm{C}\right)$ compared with those used by Liu et al. (2016). It should be noted that the larger $M_{W}$ glycates prepared in this study had much longer reaction times but gave higher $\operatorname{IgE}$ binding than the smaller $M_{W}$ glycates. Another issue with the dry heating process is the formation of advanced glycation end products, which can also generate an (unwanted) immune response (Liu et al., 2016). The aqueous method used in our study was stopped when color formation was detected. 


\section{CONCLUSIONS}

This study glycated WPI with DX of different $M_{W}$ via the initial step of the Maillard reaction and investigated the IgE binding of purified glycates using blood sera obtained from patients with CMPA. These results demonstrated that the aqueous glycation method did significantly reduce the allergenicity of whey proteins, particularly when low $M_{W}$ DX were used. Morphology results from AFM images suggested that glycation of WPI with small $M_{W}$ DX resulted in extensive protein surface coverage, including multiple bound DX. The likely reason for the observed reduction in IgE binding for our WPI-DX glycates was the loss of both linear epitopes due to glycation sites being located in their vicinity as well as modification to potential conformational epitopes due to unfolding and other structural changes to the proteins.

\section{ACKNOWLEDGMENTS}

This project was funded by the National Institute of Food and Agriculture of the US Department of Agriculture (Washington, DC). We express our sincere thanks to Christoper Tisler, Yanjie Lu, Bingqing Cai, and Shane Crowley from the University of WisconsinMadison for their valuable input into this project.

\section{REFERENCES}

Akhtar, M., and E. Dickinson. 2007. Whey protein-maltodextrin conjugates as emulsifying agents: An alternative to gum arabic. Food Hydrocoll. 21:607-616.

Böttger, F. H. 2013. Impact of Maillard modification on the in vitro digestibility and $\operatorname{IgE}$ binding capacity of whey protein isolate. MS Thesis. University of Wisconsin-Madison.

Bu, G. H., J. Lu, Z. Zheng, and Y. K. Luo. 2009. Influence of Maillard reaction conditions on the antigenicity of bovine $\alpha$-lactalbumin using response surface methodology. J. Sci. Food Agric. 89:24282434.

Bund, T., S. Allelein, A. Arunkumar, J. A. Lucey, and M. R. Etzel. 2012. Chromatographic purification and characterization of whey protein-dextran glycation products. J. Chromatogr. A 1244:98105.

Church, F. C., H. E. Swaisgood, D. H. Porter, and G. L. Catignani. 1983. Spectrophotometric assay using o-phthaldialdehyde for determination of proteolysis in milk and isolated milk proteins. J. Dairy Sci. 66:1219-1227.

Corzo-Martínez, M., A. C. Soria, J. Belloque, M. Villamiel, and F. J. Moreno. 2010. Effect of glycation on the gastrointestinal digestibility and immunoreactivity of bovine $\beta$-lactoglobulin. Int. Dairy J. 20:742-752.

de Oliveira, F. C., J. S. D. R. Coimbra, E. B. de Oliveira, A. D. G. Zuñiga, and E. E. G. Rojas. 2016. Food protein-polysaccharide conjugates obtained via the Maillard reaction: A review. Crit. Rev. Food Sci. Nutr. 56:1108-1125.

De Wit, J. N. 1990. Thermal stability and functionality of whey proteins. J. Dairy Sci. 73:3602-3612.

Dickinson, E. 2008. Interfacial structure and stability of food emulsions as affected by protein-polysaccharide interactions. Soft Matter 4:932-942.
DuBois, M., K. A. Gilles, J. K. Hamilton, P. A. Rebers, and F. Smith. 1956. Colorimetric method for determination of sugars and related substances. Anal. Chem. 28:350-356.

Dubray, G., and G. Bezard. 1982. A highly sensitive periodic acidsilver stain for 1,2-diol groups of glycoproteins and polysaccharides in polyacrylamide gels. Anal. Biochem. 119:325-329.

Dunlap, C. A., and G. L. Côté. 2005. $\beta$-Lactoglobulin-dextran conjugates: Effect of polysaccharide size on emulsion stability. J. Agric. Food Chem. 53:419-423.

Dupont, C., and D. De Boissieu. 2003. Formula feeding during cow's milk allergy. Minerva Pediatr. 55:209-216.

EFSA (European Food Safety Authority). 2004. Guidance document of the scientific panel on genetically modified organism for the risk assessment of genetically modified plants and derived food and feed. EFSA J. 99:1-94.

Ellis, R. J. 2001. Macromolecular crowding: Obvious but underappreciated. Trends Biochem. Sci. 26:597-604.

FAO/WHO (Food and Agriculture Organization of the United Nations/World Health Organization). 2001. Evaluation of allergenicity of genetically modified foods. Report of a joint FAO/WHO expert consultation on allergenicity of foods derived from biotechnology. FAO, Rome, Italy. Accessed Dec. 22, 2017. http://www.fao .org/fileadmin/templates/agns/pdf/topics/ec_jan2001.pdf.

Hattori, M. 2002. Functional improvements in food proteins in multiple aspects by conjugation with saccharides: Case studies of $\beta$-lactoglobulin-acidic polysaccharides conjugates. Food Sci. Technol. Res. 8:291-299.

Hattori, M., S. Miyakawa, Y. Ohama, H. Kawamura, T. Yoshida, K. To-o, T. Kuriki, and K. Takahashi. 2004. Reduced immunogenicity of $\beta$-lactoglobulin by conjugation with acidic oligosaccharides. J. Agric. Food Chem. 52:4546-4553.

Hattori, M., K. Nagasawa, K. Ohgata, N. Sone, A. Fukuda, H. Matsuda, and K. Takahashi. 2000. Reduced immunogenicity of $\beta$-lactoglobulin by conjugation with carboxymethyl dextran. Bioconjug. Chem. 11:84-93.

Ikeda, S. 2003. Heat-induced gelation of whey proteins observed by rheology, atomic force microscopy, and Raman scattering spectroscopy. Food Hydrocoll. 17:399-406.

Ikeda, S., and V. J. Morris. 2002. Fine-stranded and particulate aggregates of heat-denatured whey proteins visualized by atomic force microscopy. Biomacromolecules 3:382-389.

Järvinen, K.-M., P. Chatchatee, L. Bardina, K. Beyer, and H. A. Sampson. 2001. IgE and IgG binding epitopes on $\alpha$-lactalbumin and $\beta$-lactoglobulin in cow's milk allergy. Int. Arch. Allergy Immunol. 126:111-118.

Jiménez-Castaño, L., M. Villamiel, and R. López-Fandiño. 2007. Glycosylation of individual whey proteins by Maillard reaction using dextran of different molecular mass. Food Hydrocoll. 21:433-443.

Johansson, S. G. 2004. ImmunoCAP Specific IgE test: An objective tool for research and routine allergy diagnosis. Expert Rev. Mol. Diagn. 4:273-279.

Kato, A. 2002. Industrial applications of Maillard-type protein-polysaccharide conjugates. Food Sci. Technol. Res. 8:193-199.

Kobayashi, K., A. Hirano, A. Ohta, T. Yoshida, K. Takahashi, and M. Hattori. 2001. Reduced immunogenicity of $\beta$-lactoglobulin by conjugation with carboxymethyl dextran differing in molecular weight. J. Agric. Food Chem. 49:823-831.

Laemmli, U. K. 1970. Cleavage of structural proteins during the assembly of the head of bacteriophage T4. Nature 227:680-685.

Li, Z., Y. K. Luo, and L. G. Feng. 2011. Effects of Maillard reaction conditions on the antigenicity of $\alpha$-lactalbumin and $\beta$-lactoglobulin in whey protein conjugated with maltose. Eur. Food Res. Technol. 233:387-394.

Liu, F., M. Teodorowicz, M. A. J. S. van Boekel, H. J. Wichers, and K. A. Hettinga. 2016. The decrease in the IgG-binding capacity of intensively dry heated whey proteins is associated with intense Maillard reaction, structural changes of the proteins and formation of RAGE-ligands. Food Funct. 7:239-249.

Liu, G., and Q. Zhong. 2015. High temperature-short time glycation to improve heat stability of whey protein and reduce color formation. Food Hydrocoll. 44:453-460. 
Liu, J., Q. Ru, and Y. Ding. 2012. Glycation a promising method for food protein modification: Physicochemical properties and structure, a review. Food Res. Int. 49:170-183.

Lucey, J. A., M. Srinivasan, H. Singh, and P. A. Munro. 2000. Characterization of commercial and experimental sodium caseinates by multiangle laser light scattering and size-exclusion chromatography. J. Agric. Food Chem. 48:1610-1616.

Nielsen, P. M., D. Petersen, and C. Dambmann. 2001. Improved method for determining food protein degree of hydrolysis. J. Food Sci. 66:642-646.

Niemi, M., S. Jylhä, M.-L. Laukkanen, H. Söderlund, S. MäkinenKiljunen, J. M. Kallio, N. Hakulinen, T. Haahtela, K. Takkinen, and J. Rouvinen. 2007. Molecular interactions between a recombinant $\operatorname{IgE}$ antibody and the $\beta$-lactoglobulin allergen. Structure 15:1413-1421.

Nodake, Y., S. Fukumoto, M. Fukasawa, R. Sakakibara, and N. Yamasaki. 2010. Reduction of the immunogenicity of $\beta$-lactoglobulin from cow's milk by conjugation with a dextran derivative. Biosci. Biotechnol. Biochem. 74:721-726.

O'Mahony, J. A., K. P. Drapala, E. M. Mulcahy, and D. M. Mulvihill 2016. Controlled glycation of milk proteins and peptides: Functional properties. Int. Dairy J. 67:16-34.

Sampson, H. A. 2001. Utility of food-specific $\operatorname{IgE}$ concentrations in predicting symptomatic food allergy. J. Allergy Clin. Immunol. 107:891-896

Shu, Y.-W., S. Sahara, S. Nakamura, and A. Kato. 1996. Effects of the length of polysaccharide chains on the functional properties of the Maillard-type lysozyme-polysaccharide conjugate. J. Agric. Food Chem. 44:2544-2548.

Taheri-Kafrani, A., J. C. Gaudin, H. Rabesona, C. Nioi, D. Agarwal, M. Drouet, J. M. Chobert, A. K. Bordbar, and T. Haertle. 2009. Effects of heating and glycation of $\beta$-lactoglobulin on its recogni- tion by $\operatorname{IgE}$ of sera from cow milk allergy patients. J. Agric. Food Chem. 57:4974-4982.

Ter Haar, R., Y. Westphal, P. A. Wierenga, H. A. Schols, and H. Gruppen. 2011. Cross-linking behavior and foaming properties of bovine $\alpha$-lactalbumin after glycation with various saccharides. J. Agric. Food Chem. 59:12460-12466.

Wal, J.-M. 2002. Cow's milk proteins/allergens. Ann. Allergy Asthma Immunol. 89:3-10.

Wang, T., and J. A. Lucey. 2003. Use of multi-angle laser light scattering and size-exclusion chromatography to characterize the molecular weight and types of aggregates present in commercial whey protein products. J. Dairy Sci. 86:3090-3101.

Wooster, T. J., and M. A. Augustin. 2006. B-lactoglobulin-dextran conjugates: Their effect on interfacial thickness and emulsion stability. J. Colloid Interface Sci. 303:564-572.

Xu, K., and P. Yao. 2009. Stable oil-in-water emulsions prepared from soy protein-dextran conjugates. Langmuir 25:9714-9720.

Yadav, M. P., G. D. Strahan, S. Mukhopadhyay, A. T. Hotchkiss, and K. B. Hicks. 2012. Formation of corn fiber gum milk protein conjugates and their molecular characterization. Food Hydrocoll. $26: 326-333$

Zhang, M., J. Zheng, K. Ge, H. Zhang, B. Fang, L. Jiang, H. Guo, Q. Ding, and F. Ren. 2014. Glycation of $\alpha$-lactalbumin with different size saccharides: Effect on protein structure and antigenicity. Int Dairy J. 34:220-228.

Zhu, D., S. Damodaran, and J. A. Lucey. 2008. Formation of whey protein isolate (WPI)-dextran conjugates in aqueous solutions. J. Agric. Food Chem. 56:7113-7118.

Zhu, D. S. Damodaran, and J. A. Lucey. 2010. Physicochemical and emulsifying properties of whey protein isolate (WPI)-dextran conjugates produced in aqueous solution. J. Agric. Food Chem. 58:2988-2994. 